\title{
Social Learning in Birds Studied by Cross-Fostering in the Wild
}

\author{
T. Slagsvold* \\ Department of biosciences, University of Oslo, Norway
}

${ }^{*}$ Corresponding author: T. Slagsvold, Centre for Ecological and Evolutionary Synthesis (CEES), Department of Biosciences, University of Oslo, P.O. Box 1066 Blindern, N-0316 Oslo, Norway; E-mail: tore.slagsvold@ibv.uio.no

Received: August 28, 2019; Accepted: September 03, 2019; Published: September 28, 2019

\begin{abstract}
Social learning is widespread in nature and important for the behaviour, ecology and conservation of animals. We applied a method of cross-fostering between passerine birds in woodland areas in Norway, including great tits Parus major, blue tits Cyanistes caeruleus, and pied flycatchers Ficedula hypoleuca. When cross-fostering between tit species, the offspring sexually mis-imprinted on the foster species. Survival rate was similar to the controls but mating success was strongly reduced and mixed pairings occurred between cross-fostered birds of the two species, resulting in one case of hybridization. Song was affected, but the extent showed great variation among males. Male pied flycatchers raised by tits also included strophes from the foster species in their song, in particular if they had been raised together with siblings of the foster species. However, surprisingly, in flycatchers, mate choice was not affected by cross-fostering, indicating a different mechanism between song learning and mate choice. In tits, choice of nest site was affected by the crossfostering, the birds taking characteristics of the natal nest site into account, and the behaviour of members of their foster parent species. Cross-fostering had a strong effect on foraging behaviour in terms of spatial location of foraging sites and the type of prey items provided to the young. Learning foraging techniques within the natal habitat may explain why prey provided by immigrant tits new to the study area differed from those provided by local recruits. We discuss how social learning may affect the evolution, behaviour and ecology of these birds, including the evolution of nest parasitism, and how cross-fostering among species may be used in management programs of endangered species.
\end{abstract}

\section{Introduction}

During the last decades, it has been recognized that social learning is widespread in nature and strongly affects evolution, behaviour and ecology of animals, and is thus also significant for conservation [1]. Studies in captivity, with control of the rearing environment, have provided the most convincing evidence whereas less is known form the wild. We studied social learning in three hole-nesting passerine birds in woodlands in Norway, namely in the great tit Parus major, the blue tit Cyanistes caeruleus, and the pied flycatcher Ficedula hypoleuca. The species are widespread in Europe and on our study area, use nest boxes almost exclusively for breeding. The return rate of birds raised locally has been sufficiently high (5-10\% of offspring banded) to study the significance of early social learning throughout a bird's life.

The study began in 1995 in an effort to explain why only about $1 \%$ of bird species are obligate, interspecific nest parasites, although female birds are often away from the nest during the egg laying period, leaving an opportunity for females of other species to deposit an egg there to reduce their own cost of breeding. We transferred eggs between nests to simulate nest parasitism between species and this led to studies of how the cross-fostering affected social learning of several aspects of behaviour. The main results are summarized and discussed below, including how cross-fostering may be used in management programs of endangered species.

\section{Material and Methods}

We cross-fostered birds in mixed deciduous - conifer woodlands near Oslo, Norway, during 1995 - 2017. Both whole clutches and single eggs were swapped between nests to study the effect of sibling species on the degree of imprinting. All nestlings were ringed with a numbered metal ring, and adults were ringed with various combinations of plastic leg colour rings, allowing us to follow individual birds for life. Because the birds almost exclusively used our nest boxes for breeding, unringed birds were assumed to be immigrants to the study area, possibly having been raised in a different habitat [2]. The study complied with the current laws of Norway and was approved by the animal welfare committee.

\section{Results and Discussion}

\section{Mate Choice and Brood Parasitism}

The cross-fostered eggs and nestlings were accepted and cared for by their hosts, and they recruited into the adult population with a similar frequency as the controls [3,4]. However, in tits, most of the cross-fostered birds became sexually mis-imprinted, trying to mate with a member of the foster species. Therefore, many did not succeed in pairing with a conspecific but they often mated with a member of the other species that had also been cross-fostered. Over the years, more than 30 such mixed pairs were found (T. Slagsvold, 
unpublished data). Their eggs often did not hatch, but occasionally the female hatched nestlings of her own species which had resulted from extra-pair copulation with a conspecific male that was not her social partner. Only one case of mixed-pairings resulted in a hybrid (between a female great tit and a male blue tit), to our knowledge the only hybrid known between the two species.

We tested the degree of sexual mis-imprinting by presenting a live, caged bird on their territory. The cross-fostered tits typically attacked a same-sex member of the foster species much more than a conspecific rival, showing that early social learning influences intrasexual species recognition in these birds [5]. The mis-imprinting continued for life [6]. In contrast, pied flycatchers that were raised by tits did not become sexually mis-imprinted as shown by their responses to live, caged birds upon arrival in spring [3,4]. Perhaps the host species was too different in appearance, and the flycatchers overwinter in tropical Africa where the two species of tits are not found. Species recognition appears to be more innate in flycatchers than in tits [7]. Because of polygyny, many flycatchers are raised without a male present, and so an innate species recognition mechanism may have evolved to allow identification of conspecific males. In pied flycatchers, males and females usually differ in plumage colour and mate choice is very rapid, often taking only a few hours [8].

In nature, great tits and blue tits are not parasitized by other bird species. Experiments with such birds are needed to study how parasitism can start in absence of traits coevolved in an arms race with the host. We conclude that sexual imprinting may constrain the evolution of brood parasitism. It is a puzzle how some species have solved the problem $[9,10]$. However, as mentioned above, pied flycatchers do not become sexually mis-imprinted when raised by a heterospecific host. Therefore, species with an innate species recognition mechanism may more likely evolve interspecific nest parasitism.

\section{Social Dominance, Stress, Mate Guarding, Paternity and Sex Ratio}

In winter, cross-fostered tits were subordinate to conspecific controls at feeding stations although they were not smaller-sized [11]. They also seemed to suffer higher levels of stress, as measured by corticosterone in the blood, showing that rearing conditions may have long-term consequences for stress responsiveness in free-living birds [12]. Probably because of the mis-imprinting, cross-fostered male tits guarded their females less during the fertile period of their mate than did controls [13] but apparently this did not result in loss of paternity [14]. For an unknown reason, broods with at least one cross-fostered parent contained relatively more male offspring than did control broods [15].

\section{Song}

In all three species studied, cross-fostered males included strophes from the foster species when display-singing in spring. However, the variation among males was great; in the tits, some cross-fostered males only sung strophes of the foster species, some only strophes of their own species and some a mixture of the two [16,17]. Play-back studies showed that territorial cross-fostered male tits responded more strongly than controls to heterospecific song than to conspecific song [18]. Thus, social learning strongly influenced both inter- and intraspecific communication. Tits of both sexes also produced warning calls that differed according to the species they had been raised by [10]. Overall, males appear to prefer vocal tutors that visually resemble their social father although their social mother's appearance and behaviour may also influence the preference [17].

The song of pied flycatchers is much more complex than the song of tits, but even in this species, song was heavily affected by cross-fostering. If the bird had been raised both with foster parents and alongside siblings of the foster species then the strophes almost perfectly resembled those of the host species [19]. However, surprisingly, as mentioned above, mate choice in the flycatcher was not affected, indicating a different mechanism between song learning and mate choice.

\section{Choice of Nest Site}

Nest boxes were of two sizes, and most great tits used the larger ones and most blue tits the smaller. In both species, choice of nest box was affected by treatment; cross-fostered birds took the size of their natal nest box into account (vertical transmission of preference), and also the behaviour of members of their foster species (horizontal transmission of preference). However, although effects were statistically significant, the nest site choice seemed mainly to be innate [20].

\section{Foraging}

Blue tits typically forage higher above the ground, and more on twigs, than great tits, and they also feed on smaller prey. In both tit species, cross-fostering had a strong effect on the foraging and it lasted for life, the birds apparently learning from their foster parents [21]. This included both choice of foraging site [21], and which prey items they provided to the young [22]. The effect of the cross-fostering constituted about half of the difference in prey items between these birds and the controls. Cross-fostering also affected how parents chose prey items when the food demands of their offspring changed [23]

In tits, prey choice differed between immigrant and local recruits and we suggested this was a result of social learning in different natal habitats. Immigrant great tits provided more brown larvae and fewer green ones to their nestlings whereas in blue tits, a difference was found in prey size. Such foraging differences also held true within pairs, where one parent was an immigrant and the other was a local recruit, and thus when confounding factors like habitat quality, year and time of season, and the weather conditions during the videofilming were taken into account [2]. In both species, the offspring stay with their parents for about three weeks after leaving the nest and then learn foraging behaviour from their parents. The differences between the two groups of birds in prey types differed most in yearling birds. Apparently, many immigrants had been raised in different and less productive forest habitats and it took some time until they were able to forage optimally after they settled in a new habitat after natal dispersal. In great tits, immigrant females laid fewer eggs than local recruits, and 
laying date was also affected, presumably because the birds were not fully adapted to the new local micro-habitat [2].

There is great variation in feeding habits and type of parental care among bird species. For instance, precocial birds start self-feeding soon after hatching but altricial birds are characterized by a long period of parental care after hatching during which vertical transmission of skills can take place. However, learning by horizontal transmission after a bird has left its family may also be important, as demonstrated in diffusion experiments in tits [24]. We conclude that early learning is crucial for foraging behaviour later in life, and thus may affect the extent of natal dispersal, choice of habitat, and breeding success.

\section{Cross-Fostering As A Management Tool}

In birds, cross-fostering within and between species has been used to save endangered species. The case with the black robin Petroica traversi in New Zealand has been a promising example [25]. When a population is almost extinct, a few members may be brought into captivity to produce successive clutches if the eggs are removed. These extra eggs (or chicks) may then be transferred to nesting pairs of the same species in the wild (e.g. if eggs of natural nests suffer from contamination), or given to another species to rear. Here I summarize how knowledge gained from our cross-fostering studies may help such programs to succeed.

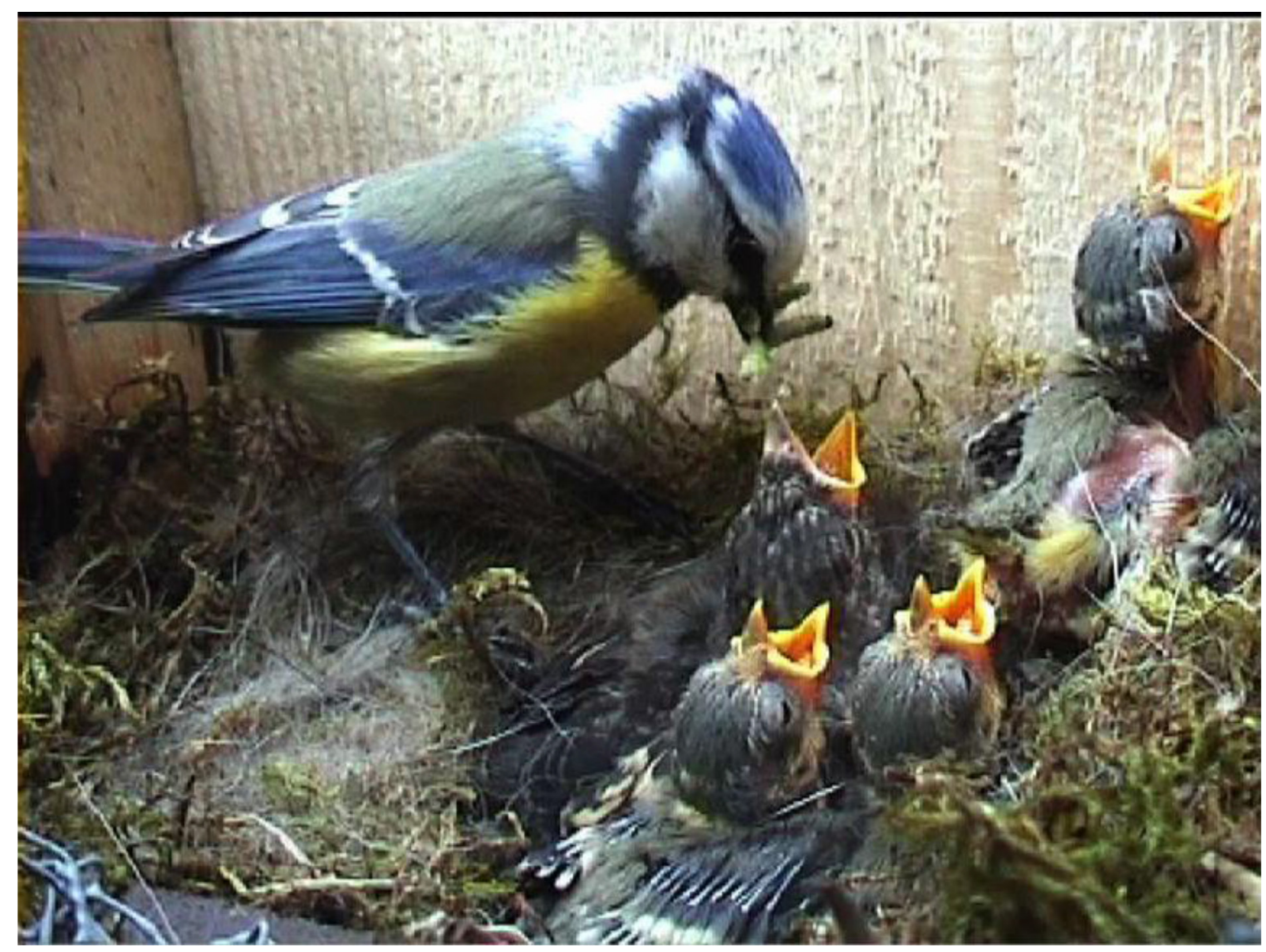

Figure 1. Male blue tit providing food to a brood containing a cross-fostered pied flycatcher chick alongside blue tit siblings. The display song of such crossfostered flycatchers included strophes typical for the host species. However, they did not become sexually mis-imprinted on the host and bred successfully with a conspecific mate.

Pied flycatcher nestlings thrived well in great tit and blue tit nests [4] but the reverse was not true. This was not because flycatcher parents rejected the tit nestlings but because the nestlings could not swallow the prey delivered by their foster parents [26]. Tit parents provide mostly soft prey items, like caterpillars, whereas flycatchers often bring adult insects harder to ingest. Therefore, great care is needed to find a suitable host species, always starting with a few temporary trials to test for negative effects. If raised together with heterospecific siblings, it is important that the cross-fostered nestlings do not hatch later than their nest mates, in particular if they are smaller and less competitive than those of the host species [9]. Thus, species with similar length of the incubation period should be preferred. Typically, obligate nest parasites in the wild are larger than their host species and have shorter incubation periods. If a smaller species is used as host in a conservation program, all host eggs should be removed to avoid suffering of host nestlings and the number of nestlings cross-fostered per host nest should also be carefully considered so parents have enough resources to rear the brood. In our study, to ensure survival, we let blue tit parents raise fewer foster great tits than would be typical of conspecific blue tit broods. Swapping of whole clutches may be 
better than swapping single eggs because of less risk of mis-imprinting when raised with conspecific siblings in the foster nests $[10,19]$.

Many of the cross-fostered tits became sexually mis-imprinted on their host species and so failed to pair with a conspecific, especially as yearlings. However, the mis-imprinting was not transferred across generations because offspring of fostered birds in the next generation showed no sign of mis-imprinting with regard to mate choice [27] and song [17]. Hence, the bottleneck of using heterospecific cross-fostering to save endangered species seemed to be the first breeding season and the first generation. When cross-fostering is applied, one should also consider a risk of hybridization. In our case, the three model species were apparently too distant genetically for this to be a problem.

Pied flycatchers raised by tits did not become sexually misimprinted. Although the males sung strophes similar to those of the foster species, they still attracted conspecific females, and some males even became polygynous [4]. Hence, cross-fostering as a management tool may be particularly useful for saving such species for which mate recognition is innate: even when members of a long-distant migrant is raised by a resident species, they may return and breed just as successfully as controls raised by their own species. We conclude that early social learning may affect the evolution, behaviour and ecology of birds, and that cross-fostering may be used with caution in management programs of endangered species.

\section{Acknowledgement}

The author is indebted to all the people that have assisted in the field during the extended study period, and to Karen Wiebe for comments on the manuscript.

\section{References}

1. Brakes P, Dall SRX, Aplin LM, Bearshop S, Carroll EL, et al. (2019) Animal cultures matter for conservation. Science 363: 1032-1034.

2. Slagsvold T, Wiebe KL (2018) Immigrants and locally recruited birds differ in prey delivered to their offspring in blue tits and great tits, Animal Behaviour 139: $127-135$.

3. Slagsvold T, Hansen BT, Johannessen LE, Lifjeld JT (2002) Mate choice and imprinting in birds studied by cross-fostering in the wild. Proceedings of the Royal Society of London B 269: 1449-1455.

4. Slagsvold T (2004) Cross-fostering of pied flycatchers (Ficedula hypoleuca) to heterospecific hosts in the wild: a study of sexual imprinting. Behaviour 141: 1079-1102.

5. Hansen BT, Slagsvold T (2003) Rival imprinting: interspecifically cross-fostered tits defend their territories against heterospecific intruders. Animal Behaviour 65: $1117-1123$.

6. Hansen BT, Johannessen LE, Slagsvold T (2008) Imprinted species recognition lasts for life in free-living great tits and blue tits. Animal Behaviour 75: 921-927.

7. Sæther SA, Sætre G-P, Borge T, Wiley C (2007) Sex chromosome-linked species recognition and evolution of reproductive isolation in flycatchers. Science 318: 95-97.

8. Dale S, Slagsvold T (1996) Mate choice on multiple cues, decision rules and sampling strategies in female pied flycatchers. Behaviour 133: 903-944.

9. Slagsvold T (1998) On the origin and rarity of interspecific nest parasitism in birds. American Naturalist 152: 28-36.

10. Slagsvold T, Hansen BT (2001) Sexual imprinting and the origin of obligate brood parasitism in birds. American Naturalist 158: 354-367.

11. Hansen BT, Slagsvold T (2004) Early learning affects social dominance: interspecifically cross-fostered tits become subdominant. Behavioral Ecology 15: $262-268$.

12. Landys MM, Goymann W, Slagsvold T (2011) Rearing conditions have longterm consequences for stress responsiveness in free-living birds. General and comparative endocrinology 174: 219-224.
13. Hansen BT, Johannessen LE, Slagsvold T (2009) Interspecific cross- fostering affects mate guarding behaviour in great tits (Parus major). Behaviour 146: 13491361 .

14. Johannessen LE, Slagsvold T, Hansen BT, Lifjeld JT (2005) Manipulation of male quality in wild tits: effects on paternity loss. Behavioral Ecology 16: 747-754.

15. Johannessen LE, Kristiansen L, Hansen BT, Slagsvold T (2009) Facultative adjustment of brood sex ratio in response to indirect manipulation of behaviour. Ethology 115: 1057-1065.

16. Johannessen LE, Slagsvold T, Hansen BT (2006) Song structure and repertoire size affected by social rearing conditions: experimental evidence from the field. Animal Behaviour 72: 83-95.

17. Eriksen A (2011) Song learning in Oscine songbirds. Tutor choice, timing, and the relationship with sexual imprinting. PhD, Department of Biosciences, University of Oslo, Norway.

18. Hansen BT, Johannessen LE, Slagsvold T (2010) Interspecific cross- fostering of great tits (Parus major) by blue tits (Cyanistes caeruleus) affects inter- and intraspecific communication. Behaviour 147: 413-424.

19. Eriksen A, Lampe H, Slagsvold T (2009) Interspecific cross-fostering affects song acquisition but not mate choice in pied flycatchers, Ficedula hypoleuca. Animal Behaviour 78: 857-863.

20. Slagsvold T, Wigdahl Kleiven K, Eriksen A, Johannessen LE (2013) Vertical and horizontal transmission of nest site preferences in titmice. Animal Behaviour 85 : 323-328.

21. Slagsvold T, Wiebe KL (2007) Learning the ecological niche. Proceedings of the Royal Society of London B 274: 19-23.

22. Slagsvold T, Wiebe KL (2011) Social learning in birds and its role in shaping a foraging niche. Philosophical Transactions of the Royal Society B 366: 969-977.

23. Wiebe KL, Slagsvold T (2015) Foraging trade-offs between prey size, delivery rate and prey type: how does niche breadth and early learning of the foraging niche affect food delivery? Ethology 121: 101-1017.

24. Aplin LM, Farine DR, Morand-Ferron J, Cockburn A, Thornton A et al. (2015) Experimentally induced innovations lead to persistent culture via conformity in wild birds. Nature 518: 538-541.

25. Butler D, Merton D (1992) The black robin. Oxford University Press, Auckland.

26. Turtumøygard T, Slagsvold T (2010) Evolution of brood parasitism in birds: constraints related to prey type. Behaviour 147: 299-317.

27. Hansen BT, Johannessen LE, Slagsvold T (2007) No cultural transmission of species recognition between parents and offspring in free-living great tits and blue tits. Behavoral Ecology and Sociobiology 61: 1203-1209.

\section{Citation:}

Tore Slagsvold (2019) Social Learning in Birds Studied by Cross-Fostering in the Wild. Integr J Vet Biosci Volume 3(2): 1-4. 